\title{
Uso do grampeador para o fechamento da faringe após laringectomia total ${ }^{1}$
}

\author{
Rogério A. Dedivitis² ${ }^{2}$ André V. Guimarães ${ }^{3}$
}

\begin{abstract}
Dedivitis RA, Guimarães AV. Uso do grampeador para o fechamento da faringe após laringectomia total. Acta Cir Bras [serial online] 2004 Jan-Fev;19(1). Disponível em URL: http://www.scielo.br/acb.

RESUMO - Objetivo: Avaliar a laringectomia total para resgate de recidiva após tratamento por radioterapia e quimioterapia concomitante por carcinoma epidermóide avançado de laringe. Métodos: Entre março de 2001 e dezembro de 2002, foram realizadas 12 laringectomias totais de resgate de recidiva com uso do grampeador linear. O protocolo de preservação de órgão para casos de câncer avançado de laringe consiste no uso de cisplatina semanal concomitante à radioterapia. A idade variou de 42 a 64 anos e onze pacientes eram do sexo masculino. Realizou-se novo estadiamento por telelaringoscopia, laringoscopia de suspensão e tomografia computadorizada. Os tumores recidivados eram restritos à endolaringe. $\mathrm{O}$ grampeador linear foi aplicado longitudinalmente na região da valécula, entre a faringe e a laringe, o mais próximo possível da asa da cartilagem tireóidea. A colocação primária de prótese fonatória foi realizada em 11 pacientes após o fechamento da faringe. Todos os pacientes obtiveram alta hospitalar entre o $4^{\circ}$ e o $7^{\circ}$ dia de pós-operatório e a dieta oral foi iniciada no $10^{\circ}$ dia. Realizou-se radiografia contrastada de faringe e esôfago entre o $21^{\circ}$ e o $30^{\circ}$ dia para avaliar a bolsa faríngea. Resultados: A aplicação do grampeador durou menos que cinco minutos. Não houve infecção, fístula faringo-cutânea, queixa de disfagia nem estreitamento ao estudo radiográfico. Os pacientes obtiveram sucesso na reabilitação fonatória a prótese. Conclusão: O uso do grampeador é tecnicamente fácil e não aumenta a incidência de fístula ou de disfagia em pacientes submetidos a laringectomia de resgate pósradioterapia. O tempo cirúrgico é reduzido e não há prejuízo na reabilitação fonatória primária com uso de prótese traqueo-esofágica.
\end{abstract}

DESCRITORES - Laringectomia. Faringe. Neoplasias laríngeas. Instrumentos cirúrgicos.

\section{Introdução}

O uso de grampeadores para o fechamento da faringe foi descrito pela primeira vez em 1969 durante a ressecção de divertículo de Zenker ${ }^{1}$. Entretanto, seu emprego durante a realização de laringectomia total ocorreu somente em $1971^{2}$. Este método permite a realização de uma laringectomia total oncologicamente adequada para tumores endolaríngeos, onde a contaminação do campo cirúrgico, por secreção de boca e faringe, seria teoricamente minimizada. Outra vantagem seria o fechamento mais rápido da faringe.

A literatura dispõe somente quatro publicações relatando o uso do grampeador linear durante o fechamento primário da faringe durante a laringectomia total ${ }^{2-5}$. A presente série estuda os resultados do método em pacientes submetidos a tratamento cirúrgico para resgate de recidiva após tratamento por radioterapia e quimioterapia concomitante por carcinoma epidermóide avançado de laringe, avaliando vantagens e desvantagens, dificuldades técnicas, a passagem primária da prótese fonatória tráqueo-esofágica e a deglutição.

\section{Métodos}

Entre março de 2001 e dezembro de 2002 , foram realizadas 12 laringectomias totais de resgate com uso do grampeador linear no Serviço de Cirurgia de Cabeça e Pescoço do Hospital Ana Costa de Santos e no Setor de Cirurgia de Cabeça e Pescoço da Irmandade da Santa Casa de Misericórdia de Santos. Os procedimentos seguiram as normas do Comitê Ético em Pesquisa da Universidade Metropolitana de Santos e estiverem de acordo com a Declaração de Helsinki de 1975.

Todos os pacientes eram portadores de carcinoma epidermóide de laringe com indicação de laringectomia total de resgate com finalidade curativa. As variáveis dos pacientes estão dispostas na Tabela 1.

Indicou-se como rotina, para os pacientes cuja indicação cirúrgica seria a laringectomia total, o protocolo de preservação de órgão com uso de cisplatina semanal, na dose de $40 \mathrm{mg} / \mathrm{m}^{2}$ concomitante a radioterapia, como primeira modalidade terapêutica. A dose de radioterapia variou entre 5800 e 7020 cGy.

1. Trabalho realizado na Disciplina de Otorrinolaringologia e Cirurgia de Cabeça e Pescoço da UNIMES, Serviço de Cirurgia de Cabeça e Pescoço do Hospital Ana Costa de Santos e Setor de Cirurgia de Cabeça e Pescoço da Irmandade da Santa Casa de Misericórdia de Santos.

2. Doutor em Medicina pelo Curso de Pós-Graduação em Otorrinolaringologia e Cirurgia de Cabeça e Pescoço da Universidade Federal de São Paulo - Escola Paulista de Medicina (UNIFESP-EPM).

3. Mestre pelo Curso de Pós-Graduação em Cirurgia de Cabeça e Pescoço do Hospital Heliópolis, São Paulo. 
TABELA 1 - Distribuição dos pacientes de acordo com os aspectos e estadiamento clínicos e o tratamento inicial realizado

\begin{tabular}{cccccc}
\hline Número & idade & Sexo & Estádio & RT & QT \\
\hline 1 & 56 & M & T3N1M0 & S & S \\
2 & 42 & M & T3N2M0 & S & S \\
3 & 58 & M & T3N1M0 & S & N \\
4 & 65 & M & T3N2M0 & S & N \\
5 & 59 & F & T4N0M0 & S & N \\
6 & 58 & M & T3N0M0 & S & S \\
7 & 64 & M & T3N0M0 & S & S \\
8 & 54 & M & T3N0M0 & S & S \\
9 & 60 & M & T3N1M0 & S & N \\
10 & 49 & M & T4N1M0 & S & N \\
11 & 51 & M & T3N1M0 & S & N \\
12 & 62 & M & T3N0M0 & S & S \\
\hline
\end{tabular}

$\mathrm{RT}=$ radioterapia Q $\mathrm{QT}=$ quimioterapia $; \mathrm{N}=$ não $\mathrm{S}=\operatorname{sim} ; \mathrm{M}=$ masculino $; \mathrm{F}=$ feminino.

Havia 14 casos de tumor residual ou recidivado de laringe. Entretanto, um não concordou com o tratamento cirúrgico e outro estava com metástases pulmonares. Entre os 12 pacientes resgatados, havia somente um do sexo feminino. Todos eram tabagistas, exceto a do sexo feminino e seis eram etilistas crônicos importantes. Em um caso a radioterapia foi interrompida aos 5.040 cGy por crescimento do tumor primário. Cinco pacientes necessitaram de traqueostomia durante o tratamento. No momento da cirurgia de resgate havia três pacientes com traqueostomia. O tempo decorrido entre a detecção da falha terapêutica e a cirurgia variou entre quatro semanas e sete meses.

Para o tratamento cirúrgico de resgate realizou-se novo estadiamento por telelaringoscopia, laringoscopia de suspensão sob anestesia geral logo antes da abordagem cirúrgica e por tomografia computadorizada. Indicamos a laringectomia com uso do grampeador somente para os casos de tumores restritos à endolaringe. Preconiza-se antibioticoterapia profilática de rotina com clindamicina e amicacina com primeira dose na indução anestésica e durante 24 horas. Após a indução anestésica, realizamos traqueostomia e laringoscopia rígida por suspensão com endoscópio de $0^{0}$ (Karl Storzâ). Tal procedimento é mandatório para confirmar se o tumor está restrito à endolaringe. Nos casos com metástase regional, iniciamos com o esvaziamento cervical radical modificado antes da dissecção da laringe, com preservação, se possível, no nervo acessório. No tempo da laringectomia, iniciamos com a secção dos músculos pré-tireoidianos junto à fúrcula esternal e separamos a glândula tireóide da traquéia através da remoção de seu istmo. Realizamos a esqueletização do osso hióide das suas inserções musculares e secção de seus cornos e dos cornos superiores da cartilagem tireóidea. Dividi-se o músculo constritor inferior da faringe, junto à asa da cartilagem tireóidea, descola-se a mucosa dos recessos piriformes e realiza-se a miotomia do cricofaríngeo. Após a transecção da traquéia, a laringe era completamente mobilizada e suspensa ventralmente, formando um ângulo de $60^{\circ}$ com plano horizontal. A faringe era mantida fechada. A borda livre da epiglote era tracionada em direção caudal através da traquéia com auxílio de pinça de Allis para não ser incluída na linha de sutura do grampeador. Neste momento, o grampeador linear TCL75 Ethiconâ foi aplicado longitudinalmente na região da valécula, entre a faringe e a laringe, estando posicionado $o$ mais próximo possível da asa da cartilagem tireóide (Figura 1). O disparo do grampeador criou uma linha de sutura dupla, separando por completo a faringe (fechada) da laringe (Figura 2). Passamos uma sonda nasogástrica para aporte calórico pós-operatório. A colocação primária de prótese fonatória Provox ${ }^{\circledR} \mathrm{n}^{\circ}$ 6 ou 8 foi realizada em 11 pacientes após o fechamento da faringe com o grampeador.

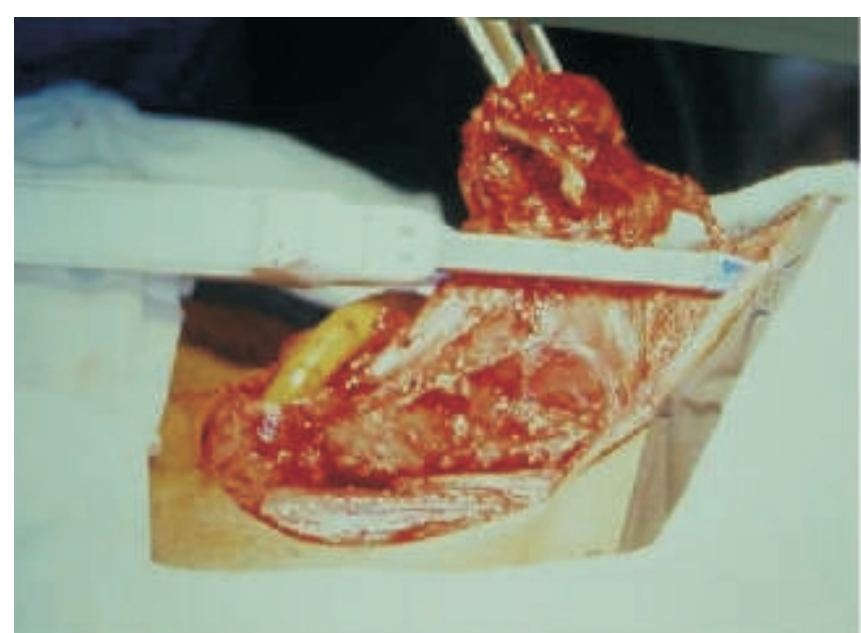

FIGURA 1 - O grampeador é posicionado o mais próximo possível da asa da cartilagem tireóide para preservação de mucosa da hipofaringe.

Todos os pacientes obtiveram alta hospitalar entre o quarto e o sétimo dia de pósoperatório. A dieta oral foi iniciada no décimo dia de pós-operatório. Realizamos radiografia contrastada de faringe e esôfago (deglutograma) entre o $21^{\circ}$ e o $30^{\circ}$ dia para avaliar a tamanho da bolsa faríngea de todos os casos.

\section{Resultados}

Não houve dificuldade técnica para preparação da laringe ou na aplicação do 


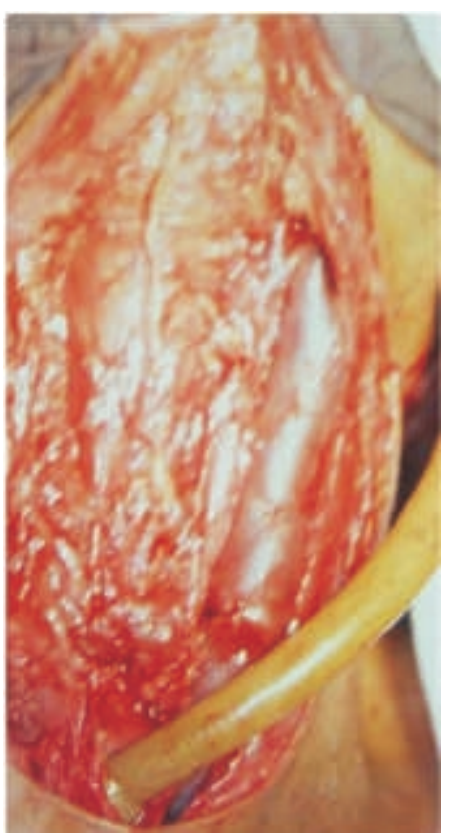

FIGURA 2 - O grampeador cria uma sutura dupla de grampos de titânio no fechamento da faringe.

grampeador. O tempo decorrido após esqueletização da laringe e da remoção da laringe com uso do grampeador foi sempre menor que cinco minutos.

Nenhum paciente apresentou sinal de infecção de ferida operatória ou manifestações clínica ou radiológica de fístula faríngea. Nenhum paciente apresentou disfagia. A radiografia contrastada da faringe e esôfago mostrou não haver sinal de estreitamentos à passagem do contraste (Figura 3).

Também não houve dificuldade técnica na passagem primária das 11 próteses fonatórias traqueo-esofágicas e todos os pacientes obtiveram sucesso na reabilitação fonatória.

\section{Discussão}

O uso do grampeador no fechamento da faringe durante a laringectomia total é fato há mais de 10 anos, todavia existe escassa literatura a respeito deste método para casos de recidiva após tratamento radioterápico. Atualmente, em todos os casos em que o emprego do grampeador em cirurgias do aparelho digestivo é possível esta tem a preferência, tanto pela economia de tempo quanto pela segurança e sua linha de sutura. $\mathrm{O}$ uso do grampeador foi ratificado para uso no trato alimentar de pacientes com divertículo de Zenker em duas grandes séries ${ }^{6,7}$, entretanto, seu uso para o fechamento da faringe é relativamente recente pelo fato de ter aplicação única em cirurgia de cabeça e pescoço. $\mathrm{O}$ fechamento da faringe após laringectomia total num tempo menor que cinco minutos é vantajoso se comparado à forma da sutura clássica da faringe com pontos separados. Além disso, não há contra-indicação, caso seja possível, de radioterapia sobre a região com os grampos. O uso do grampeador durante a laringectomia total de resgate permitiu um fechamento imediato da faringe, evitando contaminação grosseira do campo operatório e sem interferir com a reabilitação vocal à prótese traqueoesofágica. Nesta última, acreditamos também que o fato que ajudou foi ter preservado uma adequada quantidade de faringe. Há uma teoria em que o uso de sutura mecânica poderia promover a formação de fístulas, por eversão das bordas, no entanto, somente um caso de fístula em 12 pacientes operados foi observado ${ }^{5}$. Resultados semelhantes foram observados por outros autores ${ }^{3,5,9,10}$ mas, eram todos casos de resgate pós-radioterapia. Em contrapartida, há trabalho que apresenta $21 \%$ de sua casuística tratados por radioterapia prévia e sem ocorrência de fístulas ${ }^{10}$.

Há duas desvantagens em que a remoção da laringe através da técnica dita fechada com uso do grampeador não permite. A visibilização de margens e o seu recorte do paciente para estudo histopatológico. Para isto, utiliza-se a laringoscopia de suspensão prévia e, caso haja ultrapassagem do tumor dos limites da endolaringe, a técnica não é utilizada.

\section{Conclusões}

O uso do grampeador é tecnicamente fácil e não aumenta a incidência de fístula ou de disfagia em pacientes submetidos a cirurgia de resgate pós-radioterapia. Além disso, reduz o tempo cirúrgico e não interfere na reabilitação fonatória primária com uso de prótese traqueo-esofágica.

\section{Referências}

1. Hoehn JG, Payne WS. Resection of pharyngoesophageal diverticum using stapling device. Mayo Clin. Proc. 1969;44:738-41.

2. Lukyanchenko AG, Knowles JEA. Suturing of a laryngeal defect in laryngectomy. Vestn. Otorhinolaringol. 1973;33:29-30.

3. Simoncelli C, Altissimi G. Mechanical sutures of the pharynx during total laryngectomy: proposal of a closed technique. Acta Otorhinolaryngol. Ital. 1990;10:465-74.

4. Sofferman AR, Voronetsky I. Use of the linear stapler for pharyngoesofageal closure after total laringectomy. Laryngoscope 2000;110:1406-9.

5. Agrawai A, Schller DE. Closed laryngectomy using the automatic linear stapling device. Laryngoscope 2000;110:1402-5

6. Sher RL, Richtsmeier WJ. Long-term experience with endoscopic staple-assisted esophagodiverticulostomy for Zenker's diverticulum. Laryngoscope 1998;108:200-5.

7. Cultrone S, Bonarina L, Chella B, Parracchia A. Endoscopic diverticulotomy for the treatment of Zenker's diverticulum: results in 102 patients with staple-assisted endoscopy. Ann. Otol. Rhinol. Laryngol. 1999;108:810-5.

8. Sessions RB, Shemen LJ, Reuter VE. Staple closure of the gullet after laryngectomy: an experimental study. Otolaryngol. Head Neck Surg. 1986;95:491-9.

9. Talmi YP, Finkelstein Y, Gal R, Shvilli Y, Sadov R, Zohar Y. Use of a linear stapler for postlaryngectomy pharyngeal repair: a 
preliminary report. Laryngoscope 10. Westmore GA, Knowles JEA. The use of $1990 ; 100: 552-5$ stapling instrument for post-laryngectomy pharyngeal repair. J. Laryngol. Otol. 1983;97:775-8.

Dedivitis RA, Guimarães AV. The use of stapler for pharyngeal closure after total laryngectomy. Acta Cir Bras [serial online] 2004 Jan-Feb;19(1). Available from URL: http://www.scielo.br/acb.

ABSTRACT - Purpose: To evaluate total laryngectomy for the surgical salvage after a unsuccessful chemoradiation therapy treatment for advanced laryngeal squamous cell carcinoma. Methods: Twelve total laryngectomies were performed from March, 2001 to December, 2002 for recurrence salvage using the linear stapler for pharyngeal closure. The organ preservation protocol for advanced laryngeal cancer consists in weekly cysplatin concomitant to the radiation therapy. The age ranged from 42 to 64 and 11 patients were men. A new clinical stage analysis was performed with telelaryngoscopy, suspension laryngoscopy under general anesthesia and computed tomography. The recurrences should be restricted to the endolarynx. The linear stapler was applied longitudinally in the vallecula between the pharynx and larynx as close as possible to the thyroid cartilage alae. The primary placement of the phonatory prosthesis was performed in 11 patients after the pharyngeal closure. All the patients were discharged from the $4^{\text {th }}$ to the $10^{\text {th }}$ postoperative day and the oral intake was started in the $10^{\text {th }}$ postoperative day. The pharyngeal and esophageal contrasted radiography was performed from the $21^{\text {th }}$ to the $30^{\text {th }}$ postoperative day in order to evaluate the pharyngeal pouch. Results: The stapler placement delayed no more than five minutes. There was no report of infection, pharyngocutaneous fistulae, dysphagia complaint and pharyngoesophageal narrowing on the radiographic examination. The phonatory prosthesis was succfully used for the speech rehabilitation. Conclusion: The use of the stapler is technically easy to perform and it does not increase the rate of fistulae and dysphagia in patients underwent salvage laryngectomy after radiation therapy. The surgical time is reduced and the primary phonatory rehabilitation with tracheoesophageal prosthesis is feasible.

KEY WORDS - Laryngectomy. Pharynx. Laryngeal neoplasms. Surgical clips.

Conflito de interesse: nenhum Fonte de financiamento: nenhuma

Correspondência:

Rogério A. Dedivitis

Rua Olinto Rodrigues Dantas, 343/92

11050-220 Santos - SP

Tel/Fax: (13)3221-1514/3223-5550

dedivitis.hns@uol.com.br

Data do recebimento: 08/09/2003

Data da revisão: 02/10/2003

Data da aprovação: 11/11/2003 